\title{
Bundling agri-food innovations
}

\author{
Our expert panel on food value chains now shares recommendations on how to transform agri-food systems.
}

n n January 2020, Nature Sustainability and the Atkinson Center for Sustainability jointly announced the launch of the expert panel on 'Innovations to build sustainable, equitable, inclusive food value chains. Almost a year later, the report 'Socio-technical innovation bundles for agri-food system transformation' is now complete and available to our readers and the wider community.

Agri-food systems are at the heart of the relationship between people and nature. A sustainable future for all cannot be attained until agri-food systems become fully sustainable. Although these systems have gone through tremendous transformations over the past decades, increasing their capacity to sustain the life of millions of people worldwide, they are now at a critical junction. Actors operating in agri-food systems are now confronted with difficult problems such as alarming environmental degradation and the impacts of climate change, alongside public health, economic and sociocultural failures. As stated previously, the challenge ahead is huge not simply because it requires integrated knowledge about the complexity of such systems. The challenge is also huge because the new transformations needed are only possible by designing and implementing integrated solutions, and of these, unlike integrated knowledge, we still have few examples. We hope that the outcome of this expert panel will offer useful insights and suggestions to support the design of integrated solutions - socio-technical innovation bundles - for the sustainability of agri-food systems. And we hope that not only experts working on food, but also those focusing on other related, complex societal challenges will be inspired and energized by the motivations and the approach of this group of experts.

The panellists - from diverse organizations and geographies - embarked on a stimulating collaborative effort to synthesize existing knowledge on the current state of global agri-food systems, and to recognize the most critical drivers of change that could transform such systems over the next $25-50$ years. Their goal was important and ambitious: to identify the innovations that would turn agri-food systems into healthy, equitable, resilient and sustainable systems, and shed light on the enabling conditions for such innovations to diffuse and scale up.

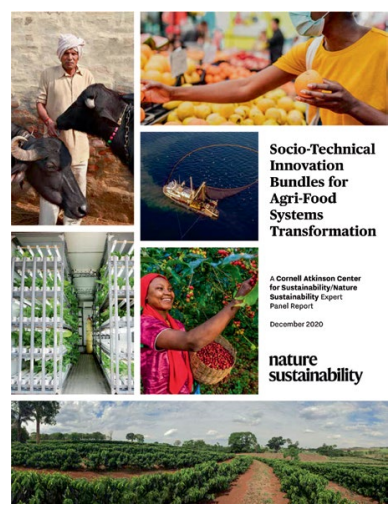

Credit: Cynthia Mathys, Cornell Atkinson Center for Sustainability.

We anticipated attention to human agency. This is reflected throughout the report and highlighted in a Comment by a group of the panellists published in this issue. Why human agency? Because very many actors in agri-food systems make decisions independently in pursuit of their own objectives and naturally most objectives end up in conflict with each other. Recognition of the critical role of human agency translated into a 'value chain' lens to assess innovations. Panellists concluded that policies and interventions aimed at reconciling differing goals and supporting cooperative behaviours along the value chain will be needed to achieve the desired transformations. But there is more to factor in. Agri-food system actors operate within diverse sociocultural, economic and biophysical settings, and with diverse realities must come diverse technological innovations. Besides, even context-specific innovations, once implemented, will have effects somewhere else in the system, and bundling them with social innovations will be crucial to address those possible effects. Clearly, as agri-food systems evolve, we won't be able to achieve progress without continuing to build knowledge that informs the development of adequate solutions, and funding support for interdisciplinary research about agri-food systems remains vital.

In the report, we read about the abundance of available technologies in domains like that of cellular and digital agriculture, food processing and safety, resource use efficiency among others. And we are also made aware that many more technologies are likely to emerge in the future. Numerous available digital innovations are of special interest as cross-cutting solutions, including, for example, applications of molecular printing, biodegradable coatings among many others. Portfolios of different innovations for different segments of the value chain will drive change. But it is not all about technologies. The report highlights the importance of developing innovative financing mechanisms. Current government farm subsidies should be reallocated to support innovations. On the private investment front, there is potential to unlock capital by shifting investors' preferences, but there are barriers. Given the diversity of agri-food systems, there should be efforts to provide research and development support where it is needed - think of Africa where most of the growth in food demand will happen over the next decades and where agri-food systems are inefficient, unsustainable and broadly speaking lag behind in terms of public health and social dimensions. There is also need of innovative social protection tools to support those who might suffer as a result of implementing disruptive innovations, and of advances in civic engagement. Rapid convergence in value chain certification schemes is critical to step up standards, and other value chain innovations are discussed in the report. Transformative innovations also have to be channelled to produce healthy and nutritious food as part of the effort to deliver systemic transformations.

So how do we ultimately drive systemic change in agri-food systems? The expert panellists here suggest bundling innovations. A socio-technical bundle is a mix of science and technology advances, properly contextualized, combined with specific institutional or policy adaptations. All actors along the agri-food value chains are vital to the success of innovations and play a role in transforming agri-food systems - our hope is that the conclusions of the expert panel report will reach out to as many of those actors as possible. Recognizing the challenge ahead, but also the immense opportunity to achieve fair, healthy and sustainable agri-food systems for all, Nature Sustainability will continue to play its part in validating and disseminating the most impactful research on the future of food.

Published online: 10 December 2020 https://doi.org/10.1038/s41893-020-00672-5 